\title{
Prostate cancer prehabilitation and the importance of multimodal interventions for person-centred care and recovery.
}

PATERSON, C., ROBERTS, C., TOOHEY, K. and MCKIE, A. 
Title: Prostate cancer prehabilitation and the importance of multimodal interventions for personcentred care and recovery

Paterson, C. (PhD, MSc, BA, PgCert LTA, FHEA, PG Non-medical prescriber, RAN), 1,2,3 Roberts, C. $(B S c)^{1,3}$, Toohey, K. (BSc, MCEP, Ph.D, PG Cert. Research, PG Cert. Tertiary Ed, AEP) ${ }^{2,3,4}$, McKie, A. $(\mathrm{BN}, \mathrm{Grad} \text { Cert renal, } \mathrm{MN})^{1,5}$

1. School of Nursing, Midwifery, and Public Health, University of Canberra, Canberra, ACT, Australia.

2. ACT Health and the Canberra Health Services, Canberra ACT, Australia

3. Prehabilitation, Activity, Cancer, Exercise and Survivorship (PACES) Research group, University of Canberra, Canberra ACT, Australia

4. Discipline of Sport and Exercise Science, Faculty of Health, University of Canberra, Canberra, ACT, Australia

5. School of Nursing, Queensland University of Technology, Brisbane, QLD, Australia

\section{Corresponding Author:}

Dr Catherine Paterson

Clinical Chair in Nursing

Faculty of Health

School of Nursing, Midwifery \& Public Health

University of Canberra ACT 2601

\&

Canberra Health Services

SYNERGY Nursing \& Midwifery Research Centre | ACT Health Directorate Level 3, Building 6, Canberra Hospital | GPO Box 825 Canberra ACT

Email: Catherine.paterson@canberra.edu.au 


\section{Abstract}

Background: Prostate cancer is the second most commonly diagnosed cancer globally. Cancer prehabilitation is defined as a process on the continuum of care that occurs between the time of a cancer diagnosis and the beginning of acute treatment. This article will discuss the importance of prostate cancer prehabilitation interventions in optimising physical and psychological recovery, to enhance person-centred care.

Data Sources: Electronic databases including CINAHL, MEDLINE, PsychINFO, Scopus, professional websites, and grey literature were searched using Google scholar.

Conclusion: Prehabilitation in cancer care aims to enhance perioperative care and recovery. An emerging field of research suggests that the preoperative period may be physically and psychologically salient to introduce modifiable self-management behaviours to optimise overall recovery.

Implications for practice: Prostate Cancer Specialist Nurses provide the hub of person-centred care across the entire cancer care continuum embedded within the multidisciplinary team. Individually tailored interventions such as exercise and pelvic floor muscle training programmes, nutritional advice, anxiety and depression reduction, and sexual well-being interventions should be considered in the prehabilitation phase of the cancer care continuum.

Key words: prostate cancer; prehabilitation; multimodal; supportive care; person centred care; nursing; multidisciplinary team 


\section{Introduction}

Prostate cancer is the second most commonly diagnosed cancer globally ${ }^{1}$. The worldwide prostate cancer burden is expected to grow to almost 2.3 million new cases by $2040^{2}$. Radical treatments for localised prostate cancer include surgery and radiotherapy which are associated with a negative impact on patients' quality of life ${ }^{3}$ and psycho-social outcomes ${ }^{4}$. Specifically, many prostate cancer survivors experience ongoing adverse side effects which include: reduced physical function, fatigue, urinary incontinence, erectile dysfunction which negatively impact quality of life and psychological well-being ${ }^{5}$. Cancer prehabilitation is defined as a process on the continuum of care that occurs between the time of a cancer diagnosis and the beginning of acute treatment ${ }^{6}$. Interventions to enhance radical therapy recovery are often introduced post-treatment in clinical practice. The main approaches tend to target erectile dysfunction and urinary incontinence through the provision of pharmacological and/or pelvic floor muscle exercises. Arguably, inadequate to address the personcentred holistic care needs ${ }^{7}$.

Prehabilitation offers a clinical route to improving the man's physical and psychological status and may buffer treatment-related deconditioning between the time of diagnosis to post-treatment recovery ${ }^{8}$, see Figure 1. Prehabilitation includes physical and psychological assessments that establish baseline functioning and identify impairments that can impact on cancer treatment-related morbidity, as well as providing targeted interventions to optimise overall well-being prior to treatment ${ }^{9}$. Patients who are active and well-functioning prior to radical therapy recuperate faster, experience better recovery and have fewer complications compared to their less fit counterparts ${ }^{8}$. Existing reviews of prehabilitation interventions describe the benefit to postoperative physical and psychological wellbeing, post-operative length of stay and surgical complications ${ }^{10,11}$. Therefore, the aim of this article is to provide an overview of prehabilitation multimodal interventions which could be used in practice to better support men affected by prostate cancer and his partner.

\section{Pelvic Floor exercises}

Radical prostatectomy (RP) is a common treatment for localised prostate cancer. Urinary incontinence is a common consequence of RP, which can cause a profound negative impact on quality of life ${ }^{12}$. Evidence synthesis on pelvic floor muscle training (PFMT) in men treated by RP is problematic due to divergence in methodologies, definitions of continence and the scheduling of the intervention ${ }^{13,14}$. However, recent clinical guidelines recommends that all men who opt for RP should have a proper assessment prior to PFMT intervention ${ }^{15}$. It is recommended, where possible, that qualified physiotherapists should ideally provide a clinical assessment and PFMT prescription for men opting for RP within the prehabilitation phase ${ }^{16}$. 


\section{Sexual well-being}

Sexual well-being is an important component of the internal fabric of human beings, as sexual wellbeing is not only concerned with the physical well-being but also the emotional, mental and social aspects of sexual health ${ }^{17,18}$. Sexual function remains an important issue in many men affected by prostate cancer and the most common treatment-related problem is erectile dysfunction ${ }^{19}$. In some men with prostate cancer, erectile dysfunction has become a larger problem than urinary incontinence and reports indicate that the level of sexual dysfunction is distressing enough to impact the individual's self-esteem and partner relationships ${ }^{20,21}$.

The treatment for erectile dysfunction can be very challenging for many health professionals, as no single treatment mode has proved more successful over another. Despite this, oral pharmacologically inhibiting phosphodiesterase type 5 (PDE5) inhibitors are traditionally the first choice of treatment 22,23 . PDE5 inhibitors are generally well-tolerated in most men however, they are contraindicated in men with diabetes, hypertension and coronary artery and in men with non-nerve sparing surgery ${ }^{24,25}$. PDE5 inhibitors do not contribute to the complete restoration of erectile function on its own, with current research indicating PDE5 inhibitors should be used in conjunction with other treatment therapies such as vacuum erection devices ${ }^{26,27}$.

Vacuum erection devices are considered an effective form of therapy for erectile dysfunction in men with prostate cancer and work best when initiated early within the rehabilitation program ${ }^{27,28}$, as part of a uniformed therapeutic regime. Although vacuum erection devices are non-invasive and highly successful, men report that they are awkward to use, time-consuming and have a high risk of penile fibrosis in long term use ${ }^{19}$. Medicated urethral suppository for erections (MUSE) is another form of treatment for erectile dysfunction in men with prostate cancer. However, due to the invasive nature and high levels of pain and discomfort reported using MUSE, it is associated with non-compliance ${ }^{29}$ and discontinuation of treatment ${ }^{30}$.

Sexual counselling has been considered an effective treatment modality for erectile dysfunction, in combination with other treatments for individuals and couples ${ }^{25,31}$. Sexual counselling aims to address the patient and their partner's anxiety and stress related to sexual well-being and support couple centred goals. Sexual counselling has associated with lower treatment discontinuation rates compared to those who did not receive sexual counselling 29,31 .

\section{Nutrition}

Nutrition has an important role in the prevention, management and survivorship of men with prostate cancer. The association between western diets and prostate cancer has been well documented, and 
includes certain nutrients such as fats, protein, carbohydrates and vitamins that have been attributed to prostate cancer disease progression at varying levels of evidence ${ }^{32-34}$. Current studies indicate a diet rich in fruit and vegetables, whilst replacing carbohydrates with whole grains and replacing red meat with fish is ideal for maintaining an appropriate body mass index in men with prostate cancer ${ }^{32,33}$. However, further high quality prospective randomised trials are needed as this area remains controversial.

Diet modification has been proven to reduce the bowel side effects of prostate cancer treatment, maintain appropriate body mass index and improve quality of life in some men ${ }^{34}$. Diet and nutritional advice is an important aspect of prehabilitation and post rehabilitation care in men with prostate cancer however, the importance of nutrition is often overlooked by health professionals ${ }^{34}$.

\section{Exercise}

Exercise has been shown to impact many systems resulting in improved function, fitness, strength, cognition and quality of life in cancer patients ${ }^{35}$. Studies suggest that exercise during treatment for cancer will reduce potentially debilitating side effects and toxicities of treatments ${ }^{36,37}$. Optimally, for sedentary men diagnosed with prostate cancer ${ }^{38}$, a referral to an experienced exercise professional at diagnosis would ensure best health related outcomes from treatment and beyond. An early referral and introduction of an exercise program prior to treatment is recommended ${ }^{39,40}$. For most patients, a prescription of moderate to high intensity aerobic and resistance-based exercise would be appropriate however, there is currently no set prescription of exercise that would be considered evidence based for all men with prostate cancer ${ }^{38}$. A referral to an exercise specialist such as an Accredited Exercise Physiologist/ Clinical Exercise Physiologist or Physical Therapist/ Physiotherapist with cancer care experience is required, as they can provide an individualised program which includes behaviour change and support and also takes into consideration any existing co-morbidities to ensure the best patient outcomes in the short and long term ${ }^{38,41}$.

\section{Psychological well-being}

Diagnosis and treatment of prostate cancer often leads to a sequalae of negative events that can have a lasting impact on a man's psychosocial well-being and overall quality of life ${ }^{42}$. It is not uncommon for these men to experience varying levels of psychological distress, which persists throughout the treatment trajectory. Traditional notions of male coping strategies indicate that men have the tendency to remain stoic in the face of adversity, as openly expressing their struggle can pose a threat to their perceived masculinity ${ }^{43,44}$. Consequently, this avoidance of emotion has been linked with reduced illness adjustment ${ }^{45,46}$ and almost one third of men have moderate to severe unmet 
psychosocial supportive care needs ${ }^{16}$. Newly diagnosed men face uncertainty about their future, with regards to disease outcome, and worry about the possibility of permanent physical limitations ${ }^{47}$. Therefore, they experience a noticeably higher incidence of anxiety and depression with reported rates of $27 \%$ and $17 \%$ respectively, which remains stable five years after treatment. Noteworthy, the increase in suicidal ideation that accompanies treatment, with a multicentre trial revealing up to $46 \%$ of men think of suicide following radical prostatectomy $y^{42,48}$. Men who adopt effective strategies to cope with cancer stressors, have the ability to self-manage challenges and have a solid foundation of social support are somewhat protected from the ill effects of their diagnosis ${ }^{46}$. However, the evidence has made it abundantly clear that 'one size does not fit all' and the unique needs of each man, along with the timing of interventions are vital planning considerations ${ }^{7,49}$. During the prehabilitation period men may be more motivated to address determinants of distress and implementing psychosocial interventions at this point can lead to an overall improvement in mood, well-being and immune function ${ }^{45,50}$.

\section{Patient Reported Outcome Measures (PRO's)}

In the clinical setting, limited time and resources are acknowledged as challenges that impact on the ability of healthcare professionals to identify and address areas of unmet needs that arise during consultation ${ }^{7,49}$. Therefore, efficiently detecting psychological distress in this population, at the point of diagnosis and beyond, is imperative for appropriate referral and timely intervention ${ }^{44}$. Validated PRO's have been supported for use in clinical practice as they provide insight into the patient experience, increase awareness of ongoing challenges, promote open-communication and lead to more-targeted self-management advice ${ }^{7}$. The Distress Thermometer (DT) ${ }^{51}$ has acceptable validity for use within the prostate cancer population. It is quick to administer and has demonstrated the ability to identify those men who require further psychological screening and assessment ${ }^{49}$. Research has shown that the DT is highly accurate in detecting distress, anxiety and depression, when assessed against other outcome measures. It may even identify men susceptible to quality of life decrements who are not captured with other standard measures. Therefore, the DT is considered a valuable addition to psychosocial care plans for men with prostate cancer and prompt timely referrals to other healthcare professionals ${ }^{44}$.

\section{Psychosocial Interventions}

There are a range of options available to support men with prostate cancer who have additional psychosocial needs. These include cognitive behavioural therapy (CBT) and cognitive behavioural stress management (CBSM), psychotherapy, psychoeducational support, peer support and selfmanagement interventions ${ }^{52-54}$. All of which can be implemented through a variety of mediums 
(online, telephone, face-to-face, individual or group delivery) ${ }^{52-54}$. Parker et al ${ }^{55}$ provided a preoperative CBSM intervention that resulted in reduced mood disturbance up to and including the day of surgery however, there was no difference between the intervention and control groups postoperatively. Penedo et al ${ }^{56}$ also employed a CBSM program which was conducted within a group setting with a peer support component. Pendeo observed that the CBSM and peer support intervention enhanced quality of life outcomes. Peer discussions can provide emotional support, experiential information exchange and reduced feelings of social isolation. A Peer support network promotes cognitive and emotional processing, which in turn facilitates disease adaptation ${ }^{52}$. Schofield et $a^{157}$ conducted a nurse-led psychoeducation group, which confirmed that group interventions could normalise experiences, bolster hope and assist with offsetting depressive symptoms and treatment related procedural concerns. However, this benefit did not translate to improvements in anxiety, unmet needs and prostate cancer specific quality of life.

As there is a strong correlation between persistent symptom burden and psychological morbidity ${ }^{58}$, evidence is emerging in favour of multimodal interventions that target physical, psychological, informational and self-management needs ${ }^{5}$. Paterson et al ${ }^{59}$ trialled a multimodal supportive care intervention (ThriverCare) and observed a statistically significant reduction in the prevalence of unmet needs for those in the intervention group. However, there was no significant difference in depression and anxiety over time. As a way forward, this study highlighted the importance of developing individualised and flexible self-management plans that recognise the specific needs of each man as they change throughout treatment process.

\section{Person-centred care}

Health care professionals can practice person-centred care by treating men affected by prostate cancer with respect, providing honest, evidence-based information, making them feel valued as individuals and working in partnership by inviting them to take part in all decision about their care and daily life. During the prehabilitation cancer trajectory and beyond, person-centred care is not a onetime event, but rather every single patient-nurse consultation should be individualised and responsive to the unique person-centred needs across the entire cancer care continuum ${ }^{60}$. Supportive care is a person-centred approach to the provision of the necessary services for those living with or affected by cancer. It meets their informational, spiritual, emotional, social, or physical needs during diagnosis, treatment, or follow-up phases including issues of health promotion and prevention, survivorship, palliation and bereavement, see Figure $2^{16}$. The prostate cancer model of consultation provides a conceptual framework which nurses can use in practice to ensure person-centred care across the entire cancer care continuum. Each member of the multidisciplinary team has defined roles in 
providing optimal care and treatment but as a collective team their contributions fit together and complement care delivery, like a jigsaw puzzle, tailored to the individual man and his partner. The Prostate Cancer Specialist Nurse acts as the "hub" along the cancer care continuum ${ }^{7}$ embedded within the MDT.

\section{Conclusion}

Oncology nurses provide a key role in the development of prehabilitation person-centred care delivery. Prehabilitation is yet to be embedded as standard care practice however, emerging evidence conducted in prehabilitation interventions appears to have merit for men affected by prostate cancer. Additional research is needed to assess the longer-term physical and psychological outcomes of multimodal prehabilitation interventions in men affected by prostate cancer and their partners. 


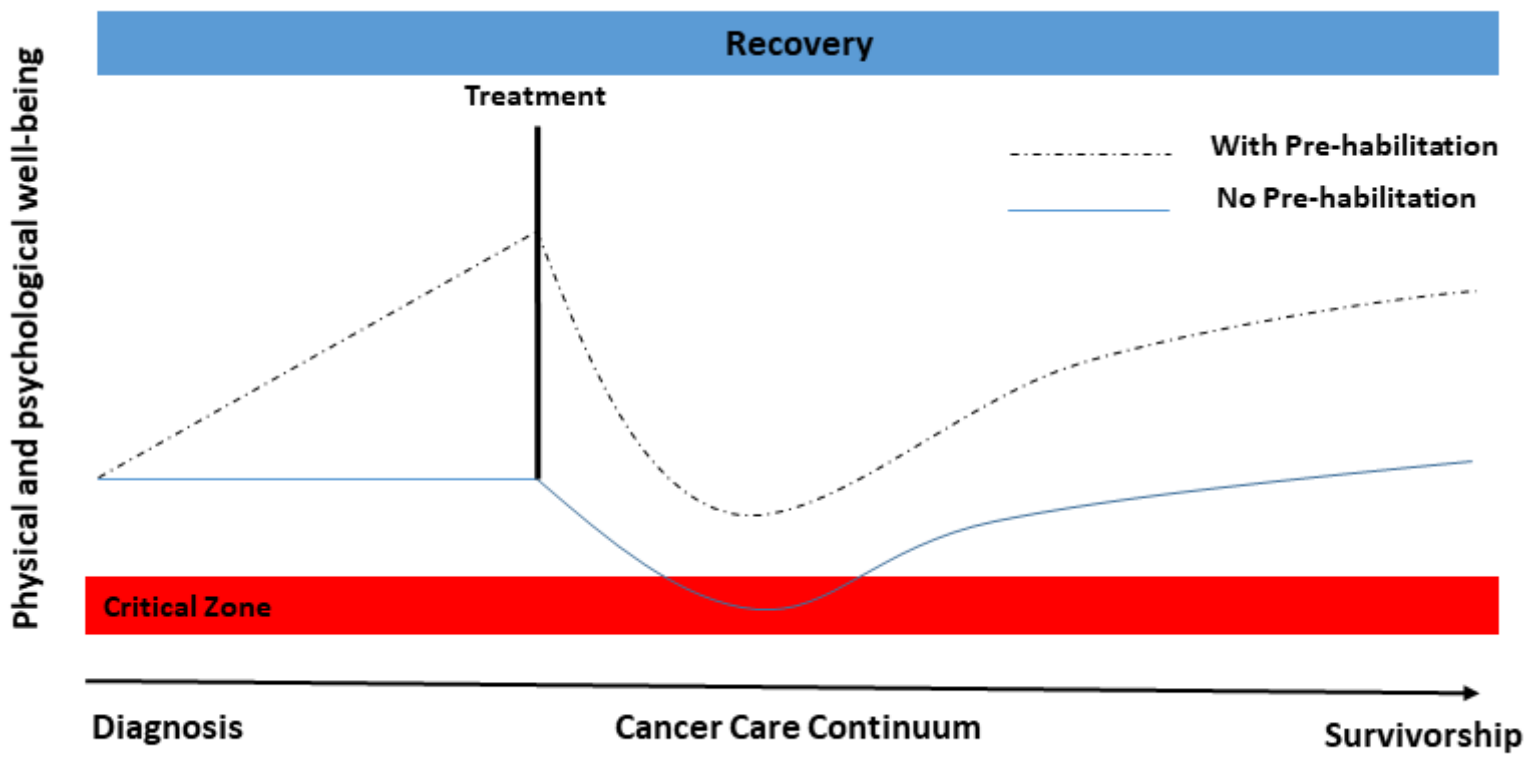

Figure 1. Hypothesized mechanism of prehabiliation supportive care interventions on patient outcomes 


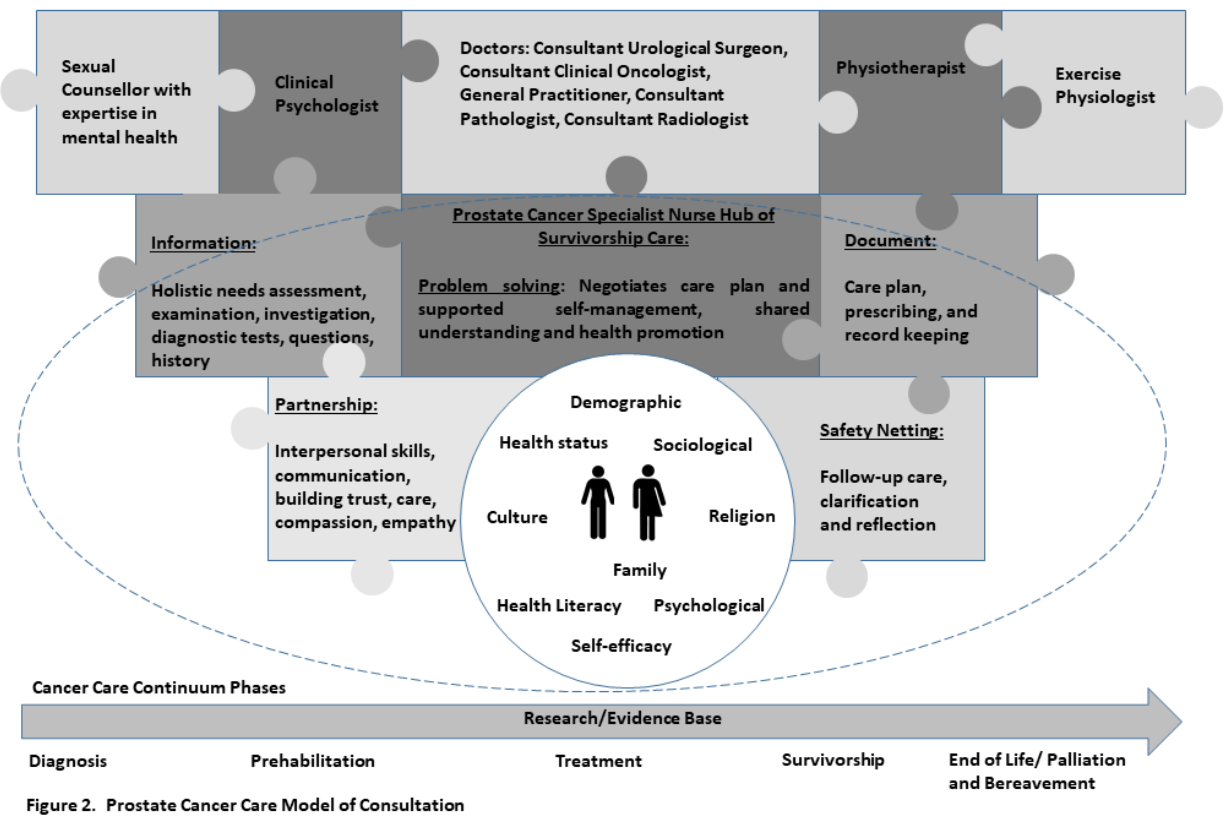




\section{References}

1. Culp MB, Soerjomataram I, Efstathiou JA, Bray F, Jemal A. Recent Global Patterns in Prostate Cancer Incidence and Mortality Rates. European Urology. 2020;77(1):38-52.

2. Ferlay J, Ervik M, Lam F, et al. Global cancer observatory: cancer today. Lyon, France: International Agency for Research on Cancer. 2018.

3. Sureda A, Fumadó L, Ferrer M, et al. Health-related quality of life in men with prostate cancer undergoing active surveillance versus radical prostatectomy, external-beam radiotherapy, prostate brachytherapy and reference population: a cross-sectional study. Health and quality of life outcomes. 2019;17(1):1-9.

4. Paterson C, Jones M, Rattray J, Lauder W. Exploring the relationship between coping, social support and health-related quality of life for prostate cancer survivors: a review of the literature. European Journal of Oncology Nursing. 2013;17(6):750-759.

5. Paterson C, Primeau C, Pullar I, Nabi G. Development of a prehabilitation multimodal supportive care interventions for men and their partners before radical prostatectomy for localized prostate cancer. Cancer nursing. 2019;42(4):E47-E53.

6. Molenaar CJ, Papen-Botterhuis NE, Herrle F, Slooter GD. Prehabilitation, making patients fit for surgery-a new frontier in perioperative care. Innovative Surgical Sciences. 2019;4(4):132138.

7. Paterson C, Nabi G. A model of consultation in prostate cancer care: Evidence from a systematic review. Cancer nursing. 2017;40(4):276-288.

8. Knowles C, DunneJ D, Ashcroft J, et al. Prehab matters-a prehabilitation service for cancer patients undergoing major abdominal surgery. Physiotherapy. 2019;105:e132.

9. Carli F, Silver JK, Feldman LS, et al. Surgical prehabilitation in patients with cancer: state-ofthe-science and recommendations for future research from a panel of subject matter experts. Physical Medicine and Rehabilitation Clinics. 2017;28(1):49-64.

10. Moran J, Guinan E, McCormick P, et al. The ability of prehabilitation to influence postoperative outcome after intra-abdominal operation: a systematic review and metaanalysis. Surgery. 2016;160(5):1189-1201.

11. Tsimopoulou I, Pasquali S, Howard R, et al. Psychological prehabilitation before cancer surgery: a systematic review. Annals of surgical oncology. 2015;22(13):4117-4123.

12. Jones CP, Campbell JG, Broghammer JA. Post-Prostatectomy Incontinence: an Update on Current Management. Current Bladder Dysfunction Reports. 2019;14(4):256-265.

13. Chang JI, Lam V, Patel MI. Preoperative Pelvic Floor Muscle Exercise and Postprostatectomy Incontinence: A Systematic Review and Meta-analysis. Eur Urol. 2016;69(3):460-467.

14. Strączyńska A, Weber-Rajek M, Strojek K, et al. The Impact Of Pelvic Floor Muscle Training On Urinary Incontinence In Men After Radical Prostatectomy (RP) - A Systematic Review. Clin Interv Aging. 2019;14:1997-2005.

15. Sandhu JS, Breyer B, Comiter C, et al. Incontinence after Prostate Treatment: AUA/SUFU Guideline. J Urol. 2019;202(2):369-378.

16. Paterson C, Robertson A, Smith A, Nabi G. Identifying the unmet supportive care needs of men living with and beyond prostate cancer: a systematic review. European Journal of Oncology Nursing. 2015;19(4):405-418.

17. O'Connor SR, Connaghan J, Maguire R, et al. Healthcare professional perceived barriers and facilitators to discussing sexual wellbeing with patients after diagnosis of chronic illness: A mixed-methods evidence synthesis. Patient Educ Couns. 2019;102(5):850-863.

18. World Health Organisation. Defining Sexual Health. https://www.who.int/reproductivehealth/publications/sexual_health/defining_sh/en/. Published 2020. Accessed 02.05.2020. 
19. Chung E, Brock G. Sexual rehabilitation and cancer survivorship: a state of art review of current literature and management strategies in male sexual dysfunction among prostate cancer survivors. J Sex Med. 2013;10 Suppl 1:102-111.

20. Naccarato A, Consuelo Souto S, Matheus WE, Ferreira U, Denardi F. Quality of life and sexual health in men with prostate cancer undergoing radical prostatectomy. The Aging Male. 2018:1-8.

21. Mehta A, Pollack CE, Gillespie TW, et al. What Patients and Partners Want in Interventions That Support Sexual Recovery After Prostate Cancer Treatment: An Exploratory Convergent Mixed Methods Study. Sex Med. 2019;7(2):184-191.

22. McConkey R. Effect of erectile dysfunction following prostate cancer treatment. Nurs Stand. 2015;30(12):38-44.

23. Andersson KE. PDE5 inhibitors - pharmacology and clinical applications 20 years after sildenafil discovery. Br J Pharmacol. 2018;175(13):2554-2565.

24. Pahlajani G, Raina R, Jones S, Ali M, Zippe $C$. Vacuum erection devices revisited: its emerging role in the treatment of erectile dysfunction and early penile rehabilitation following prostate cancer therapy. J Sex Med. 2012;9(4):1182-1189.

25. Le TV, Tsambarlis P, Hellstrom WJG. Pharmacodynamics of the agents used for the treatment of erectile dysfunction. Expert Opin Drug Metab Toxicol. 2019;15(2):121-131.

26. Clavell-Hernández J, Wang R. PDE-5 inhibitors should be used post radical prostatectomy as erection function rehabilitation? | Opinion: No. Int Braz J Urol. 2017;43(3):390-393.

27. Chung E, Gillman M. Prostate cancer survivorship: a review of erectile dysfunction and penile rehabilitation after prostate cancer therapy. Medical Journal of Australia. 2014;200(10):582585.

28. Yang X-L, Yang Y, Fu F-D, Wu C-J, Qin F, Yuan J-H. Optimal pressure in penile rehabilitation with a vacuum erection device: evidence based on a rat model. Asian journal of andrology. 2019;21(5):516.

29. Saleh A, Abboudi H, Ghazal-Aswad M, Mayer EK, Vale JA. Management of erectile dysfunction post-radical prostatectomy. Res Rep Urol. 2015;7:19-33.

30. Sopko NA, Burnett AL. Erection rehabilitation following prostatectomy--current strategies and future directions. Nat Rev Urol. 2016;13(4):216-225.

31. Katz A. Counseling men with prostate cancer and their partners. Sexual and Relationship Therapy. 2016;31(4):473-487.

32. Lin PH, Aronson W, Freedland SJ. An update of research evidence on nutrition and prostate cancer. Urol Oncol. 2019;37(6):387-401.

33. Matsushita M, Fujita K, Nonomura N. Influence of Diet and Nutrition on Prostate Cancer. Int J Mol Sci. 2020;21(4):1447.

34. McLaughlin K, Hedden L, Pollock P, Higano C, Murphy RA. Assessing the nutritional needs of men with prostate cancer. Nutrition Journal. 2019;18(1):81.

35. Papadopoulos E, Mina DS, Culos-Reed N, et al. Effects of six months of aerobic and resistance training on metabolic markers and bone mineral density in older men on androgen deprivation therapy for prostate cancer. Journal of Geriatric Oncology. 2020.

36. Cormie P, Galvão DA, Spry N, et al. Can supervised exercise prevent treatment toxicity in patients with prostate cancer initiating androgen-deprivation therapy: a randomised controlled trial. BJU International. 2015;115(2):256-266.

37. Galvão DA, Spry N, Denham J, et al. A Multicentre Year-long Randomised Controlled Trial of Exercise Training Targeting Physical Functioning in Men with Prostate Cancer Previously Treated with Androgen Suppression and Radiation from TROG 03.04 RADAR. European Urology. 2014;65(5):856-864.

38. Hayes SC, Newton RU, Spence RR, Galvão DA. The Exercise and Sports Science Australia position statement: Exercise medicine in cancer management. Journal of Science and Medicine in Sport. 2019;22(11):1175-1199. 
39. Mundell L, Daly R, Macpherson H, Fraser S. Cognitive decline in prostate cancer patients undergoing ADT: a potential role for exercise training. Endocrine-Related Cancer. 2017;24(4):R145-R155.

40. Newton RU, Galvão DA, Spry N, et al. Timing of exercise for muscle strength and physical function in men initiating ADT for prostate cancer. Prostate Cancer and Prostatic Diseases. 2020.

41. Cormie P, Atkinson M, Bucci L, et al. Clinical Oncology Society of Australia position statement on exercise in cancer care. Medical Journal of Australia. 2018;209(4):184-187.

42. Vartolomei L, Shariat SF, Vartolomei MD. Psychotherapeutic Interventions Targeting Prostate Cancer Patients: A Systematic Review of the Literature. European urology oncology. 2018;1(4):283-291.

43. Wall DP, Kristjanson LJ, Fisher C, Boldy D, Kendall GE. Responding to a diagnosis of localized prostate cancer: men's experiences of normal distress during the first 3 postdiagnostic months. Cancer nursing. 2013;36(6):E44-E50.

44. Chambers SK, Zajdlewicz L, Youlden DR, Holland JC, Dunn J. The validity of the distress thermometer in prostate cancer populations. Psycho-Oncology. 2014;23(2):195-203.

45. Orom H, Nelson CJ, Underwood III W, Homish DL, Kapoor DA. Factors associated with emotional distress in newly diagnosed prostate cancer patients. Psycho-Oncology. 2015;24(11):1416-1422.

46. Spendelow JS, Joubert HE, Lee H, Fairhurst BR. Coping and adjustment in men with prostate cancer: a systematic review of qualitative studies. Journal of Cancer Survivorship. 2018;12(2):155-168.

47. Paterson C, Jones M, Rattray J, Lauder W, Nabi G. What is the mechanism effect that links social support to coping and psychological outcome within individuals affected by prostate cancer? Real time data collection using mobile technology. European Journal of Oncology Nursing. 2016;21:126-133.

48. Bourke L, Boorjian SA, Briganti A, et al. Survivorship and improving quality of life in men with prostate cancer. European urology. 2015;68(3):374-383.

49. Lotfi-Jam K, Gough K, Schofield P, Aranda S. Profile and predictors of global distress: Can the DT guide nursing practice in prostate cancer? Palliative \& supportive care. 2014;12(1):5-14.

50. Treanor C, Kyaw T, Donnelly M. An international review and meta-analysis of prehabilitation compared to usual care for cancer patients. Journal of Cancer Survivorship. 2018;12(1):6473.

51. Roth AJ, Kornblith AB, Batel-Copel L, Peabody E, Scher HI, Holland JC. Rapid screening for psychologic distress in men with prostate carcinoma: a pilot study. Cancer: Interdisciplinary International Journal of the American Cancer Society. 1998;82(10):1904-1908.

52. Chambers SK, Pinnock C, Lepore SJ, Hughes S, O'Connell DL. A systematic review of psychosocial interventions for men with prostate cancer and their partners. Patient Education and Counseling. 2011;85(2):e75-e88.

53. Cockle-Hearne J, Faithfull S. Self-management for men surviving prostate cancer: a review of behavioural and psychosocial interventions to understand what strategies can work, for whom and in what circumstances. Psycho-oncology. 2010;19(9):909-922.

54. Parahoo K, McDonough S, McCaughan E, et al. Psychosocial interventions for men with prostate cancer: a Cochrane systematic review. BJU international. 2015;116(2):174-183.

55. Parker PA, Pettaway CA, Babaian RJ, et al. The effects of a presurgical stress management intervention for men with prostate cancer undergoing radical prostatectomy. Journal of Clinical Oncology. 2009;27(19):3169.

56. Penedo FJ, Molton I, Dahn JR, et al. A randomized clinical trial of group-based cognitivebehavioral stress management in localized prostate cancer: development of stress management skills improves quality of life and benefit finding. Annals of Behavioral Medicine. 2006;31(3):261-270. 
57. Schofield P, Gough K, Lotfi-Jam K, et al. Nurse-led group consultation intervention reduces depressive symptoms in men with localised prostate cancer: a cluster randomised controlled trial. BMC cancer. 2016;16(1):637.

58. Watson E, Shinkins B, Frith E, et al. Symptoms, unmet needs, psychological well-being and health status in survivors of prostate cancer: implications for redesigning follow-up. BJU international. 2016;117(6B):E10-E19.

59. Paterson C, Primeau C, Nabi G. A pilot randomised controlled trial of a multimodal supportive care (ThriverCare) intervention for managing unmet supportive care needs in men with metastatic prostate cancer on hormonal treatment and their partner/caregivers. European Journal of Oncology Nursing. 2018;37:65-73.

60. McCormack B, Dewing J, McCance T. Developing person-centred care: addressing contextual challenges through practice development. 2011. 